\title{
Duality and existence for a class of mass transportation problems and economic applications
}

\author{
G. Carlier *
}

14th May 2002

\begin{abstract}
We establish duality, existence and uniqueness results for a class of mass transportations problems. We extend a technique of W. Gangbo [9] using the Euler Equation of the dual problem. This is done by introducing the $h$-Fenchel Transform and using its basic properties. The cost functions we consider satisfy a generalization of the so-called Spence-Mirrlees condition which is well-known by economists in dimension 1. We therefore end this article by a somehow unexpected application to the economic theory of incentives.
\end{abstract}

\section{Résumé}

Nous établissons dans cet article des résultats de dualité, d'existence et d'unicité pour une classe de problèmes de transport optimal de masse. La nouveauté réside ici dans l'emploi de la transformée de Fenchel h-convexe qui permet d'utiliser un argument de W. Gangbo [9] consistant à exploiter l'équation d'Euler du problème dual. Les coûts de transport que nous considérons satisfont une condition généralisant la condition de Spence-Mirrlees bien connue des économistes en dimension 1. Nous terminons ainsi cet article par une application de notre résultat à la théorie économique des incitations.

JEL Classification: C61, D82.

Mathematics Subject Classification 2000: 90C08, 90C46, $91 \mathrm{~B} 40$.

${ }^{*}$ Université Bordeaux 1, MAB, UMR CNRS 5466 and Université Bordeaux IV, GRAPE, UMR CNRS 5113, Avenue Léon Duguit, 33608, Pessac, FRANCE Guillaume.Carlier@math.u-bordeaux.fr. 


\section{Introduction and main statement}

\subsection{Assumptions and notations}

Let us first recall that, given a probability space $\left(\Omega_{1}, \mathcal{A}_{1}, \mu_{1}\right)$, a measurable space $\left(\Omega_{2}, \mathcal{A}_{2}\right)$ and a measurable map $f: \Omega_{1} \rightarrow \Omega_{2}$, the push-forward of $\mu_{1}$ through $f$, denoted $f \sharp \mu_{1}$ is the probability measure on $\left(\Omega_{2}, \mathcal{A}_{2}\right)$ defined by:

$$
f \sharp \mu_{1}(B):=\mu_{1}\left(f^{-1}(B)\right)
$$

for every $B \in \mathcal{A}_{2}$.

In all the following, $\Omega$ is some bounded connected open subset of $\mathbb{R}^{n}$, and $\mu$ is some probability measure in $\Omega$ which is absolutely continuous with respect to the $n$-dimensional Lebesgue measure, with a positive Radon-Nikodym derivative with respect to the $n$-dimensional Lebesgue measure and such that $\mu(\partial \Omega)=0$.

We are also given a compact Polish space $Y$, a Radon probability measure $\nu$ on $Y$ and a function $h: \bar{\Omega} \times Y \rightarrow \mathbb{R}$ which satisfies:

$$
h \in C^{0}(\bar{\Omega} \times Y, \mathbb{R}),
$$

for every $\omega \subset \subset \Omega$ there exists $c(\omega)>0$ such that for all $\left(x_{1}, x_{2}\right) \in \omega^{2}$

$$
\sup _{y \in Y}\left|h\left(x_{1}, y\right)-h\left(x_{2}, y\right)\right| \leq c(\omega)\left\|x_{1}-x_{2}\right\|,
$$

for all $y \in Y, h(., y)$ is differentiable in $\Omega$ and for all $\left(y_{1}, y_{2}, x\right) \in Y^{2} \times \Omega$

$$
\frac{\partial h}{\partial x}\left(x, y_{1}\right)=\frac{\partial h}{\partial x}\left(x, y_{2}\right) \Rightarrow y_{1}=y_{2}
$$

Assumption (3) plays an important role in the proofs and we shall see that it may be interpreted as a generalization of the well-known one of Spence and Mirrlees, this assumption was first introduced by Levin in [13].

Our aim is to study the following Monge's mass transportation problem:

$$
(\mathcal{M}) \sup _{s \in \Delta(\mu, \nu)} J(s):=\int_{\Omega} h(x, s(x)) d \mu(x)
$$

with:

$$
\Delta(\mu, \nu):=\{s \text { is a Borel map : } \Omega \rightarrow Y \text { s.t. } s \sharp \mu=\nu\} .
$$

The associated Monge-Kantorovich problem is the linear (relaxation of $(\mathcal{M}))$ program: 


$$
(\mathcal{M K}) \sup _{\gamma \in \Gamma(\mu, \nu)} K(\gamma):=\int_{\Omega \times Y} h(x, y) d \gamma(x, y)
$$

with:

$\Gamma(\mu, \nu):=\left\{\gamma\right.$ is a Borel probabiliy measure on $\Omega \times Y$ s.t. $\left.\pi_{1} \sharp \gamma=\mu, \pi_{2} \sharp \gamma=\nu\right\}$

where $\pi_{1}(x, y)=x, \pi_{2}(x, y)=y$ for all $(x, y) \in \Omega \times Y$.

Finally, we define the (dual of $(\mathcal{M})$ ) problem:

$$
(\mathcal{D}) \inf _{(\psi, \phi) \in E_{h}} L(\psi, \phi):=\int_{\Omega} \psi d \mu+\int_{Y} \phi d \nu
$$

with:

$E_{h}:=\{(\psi, \phi)$, real-valued measurable s.t. $\psi(x)+\phi(y) \geq h(x, y), \forall(x, y) \in \Omega \times Y\}$.

\subsection{Main result}

If $\psi$ is a given real-valued function defined on $\Omega$, we define the $h$-Fenchel Transform of $\psi, \psi^{h}$ by:

$$
\psi^{h}(y):=\sup _{x \in \Omega} h(x, y)-\psi(x), \text { for all } y \in Y
$$

In a similar way, if $\phi$ is a given real-valued function defined on $Y$, we define the $\breve{h}$-Fenchel Transform of $\phi, \phi^{\check{h}}$ by:

$$
\phi^{\check{h}}(x):=\sup _{y \in Y} h(x, y)-\phi(y), \text { for all } x \in \Omega .
$$

Our main result can then be stated as follows:

Theorem 1 Under assumptions (1), (2), (3) the following assertions hold:

1) problems $(\mathcal{M}),(\mathcal{M K})$ and $(\mathcal{D})$ admit at least one solution,

2) $(\mathcal{D})$ is dual to $(\mathcal{M})$ and $(\mathcal{M K})$ in the sense:

$$
\inf (\mathcal{D})=\sup (\mathcal{M})=\sup (\mathcal{M K}),
$$

3) the minimum in $(\mathcal{D})$ is attained by a pair $(\bar{\psi}, \bar{\phi})$ such that:

$$
\bar{\psi}=\bar{\phi}^{\check{h}}, \bar{\phi}=\bar{\psi}^{h}
$$


there exists moreover some Borel map $\bar{s}$ from $\Omega$ to $Y$ which satisfies:

$$
\bar{\psi}(x)+\bar{\phi}(\bar{s}(x))=h(x, \bar{s}(x)), \text { for all } x \in \Omega,
$$

$\bar{s} \in \Delta(\mu, \nu)$ and is a solution of $(\mathcal{M})$, and $(i d, \bar{s}) \sharp \mu$ is a solution of $(\mathcal{M K})$,

4) uniqueness also holds: if $s$ is a solution of $(\mathcal{M})$ then $s=\bar{s} \mu$-a.e., $(i d, \bar{s}) \sharp \mu$ is the unique solution of $(\mathcal{M K})$, and if $(\psi, \phi)$ is a solution of $(\mathcal{D})$ then $\psi-\bar{\psi}$ (respectively $\phi-\bar{\phi})$ is equal to some constant $\mu$-a.e. (respectively v-a.e.).

In Section 2, technical lemmas are established and basic properties of the $h$-Fenchel transform are proved. In Section 3, the main result is proved. Finally, in Section 4, we adress a question arising in the economic theory of incentives and show how assumption (3) can be interpreted as a natural generalization of the Spence-Mirrlees condition. In this framework, our main result enables to prove a general re-allocation principle.

The problem of optimal measure preserving maps $(\mathcal{M})$ has received a lot of attention since related questions naturally arise in fluid mechanics [2], differential geometry (see [16] for relation with a classical result of Aleksandrov [1]), shape optimization [4], functional analysis [11], [12], probability [19] and economics. In the case $Y \subset \mathbb{R}^{n}$ and $h(x, y)=x \cdot y$, the problem was solved by Brenier [3] who proved the important Polar Factorization Theorem and existence and uniqueness of an optimal map which is the gradient of some convex potential. This result was then extended by Mc Cann and Gangbo [10] for costs of the form $c(x-y)$ with $c$ strictly convex. The result stated in Theorem 1, is very much in that spirit since it expresses existence and uniqueness of an optimal allocation map which is a measurable selection of the $h$-subdifferential of some $h$-convex potential. Similar characterization results were obtained by $\mathrm{V}$. Levin [13] using a different approach based on cyclical monotonicity and the relaxed problem $(\mathcal{M K})$.

\section{Technical preliminaries and $h$-Fenchel Trans- form}

In what follows $\psi$ will always denote some function $: \Omega \rightarrow \mathbb{R} \cup\{+\infty\}$ and $\phi$ some function $: Y \rightarrow \mathbb{R} \cup\{+\infty\}$.

Definition 1 1) $\psi$ is h-convex if and only if there exists a nonempty subset A of $Y \times \mathbb{R}$ such that:

$$
\psi(x)=\sup _{(y, t) \in A} h(x, y)+t, \text { for all } x \in \Omega .
$$


2) $\phi$ is $\breve{h}$-convex if and only if there exists a nonempty subset $B$ of $\Omega \times \mathbb{R}$ such that:

$$
\phi(y)=\sup _{(x, t) \in B} h(x, y)+t, \text { for all } y \in Y .
$$

Remark. If $\psi$ is $h$-convex then either $\psi$ is identically $+\infty$ or it is bounded. Note also that finite $\breve{h}$-convex potentials are l.s.c, hence $\nu$-measurable.

Definition 2 1) The h-Fenchel Transform of $\psi, \psi^{h}$, is the $\breve{h}$-convex function defined by:

$$
\psi^{h}(y):=\sup _{x \in \Omega} h(x, y)-\psi(x), \text { for all } y \in Y .
$$

2) The $\check{h}$-Fenchel Transform of $\phi, \phi^{\check{h}}$ is the $h$-convex function defined by:

$$
\phi^{\check{h}}(x):=\sup _{y \in Y} h(x, y)-\phi(y), \text { for all } x \in \Omega .
$$

Obviously, Young's inequalities hold:

$$
\psi(x)+\psi^{h}(y) \geq h(x, y), \text { for all }(x, y) \in \Omega \times Y
$$

and:

$$
\phi^{\breve{h}}(x)+\phi(y) \geq h(x, y), \text { for all }(x, y) \in \Omega \times Y .
$$

\section{Proposition 1}

$$
\begin{gathered}
\left(\psi^{h}\right)^{\check{h}}(x)=\sup \{f(x): f \leq \psi, f \text { is } h \text {-convex }\}, \text { for all } x \in \Omega, \\
\left(\phi^{\check{h}}\right)^{h}(y)=\sup \{g(x): g \leq \phi, f \text { is } \check{h} \text {-convex }\}, \text { for all } y \in Y .
\end{gathered}
$$

It follows that $\psi$ (respectively $\phi)$ is $h$-convex (respectively $\check{h}$-convex) if and only if $\psi=\left(\psi^{h}\right)^{\check{h}}$ (respectively $\left.\phi=\left(\phi^{\check{h}}\right)^{h}\right)$.

\section{Proof.}

First $\left(\psi^{h}\right)^{\check{h}}$ is $h$-convex and Young's inequality yields $\left(\psi^{h}\right)^{\check{h}} \leq \psi$ so that, if we define:

$$
V(x):=\sup \{f(x): f \leq \psi, f \text { is } h \text {-convex }\}, \text { for all } x \in \Omega,
$$

then :

$$
\left(\psi^{h}\right)^{\check{h}} \leq V \leq \psi .
$$

Since $V$ is $h$-convex, there exists a nonempty subset $A$ of $Y \times \mathbb{R}$ such that:

$$
V(x)=\sup _{(y, t) \in A} h(x, y)+t, \text { for all } x \in \Omega .
$$


Let $\left(y_{0}, t_{0}\right) \in A$ and $\xi:=h\left(., y_{0}\right)+t_{0}$ we have:

$$
\psi \geq \xi \Rightarrow\left(\psi^{h}\right)^{\check{h}} \geq\left(\xi^{h}\right)^{\check{h}}
$$

of course $\xi \geq\left(\xi_{\breve{h}}^{h}\right)^{\check{h}}$ and since $\xi^{h}\left(y_{0}\right)=-t_{0}$ then $\left(\xi^{h}\right)^{\breve{h}}(x) \geq h\left(x, y_{0}\right)+t_{0}=\xi(x)$ for all $x$ so $\left(\xi^{h}\right)^{\breve{h}}=\xi$, with (8) we get $\left(\psi_{\breve{h}}^{h}\right)^{\breve{h}} \geq \xi$ and since $\left(y_{0}, t_{0}\right)$ is arbitrary in $A$ taking the supremum yields $\left(\psi^{h}\right)^{\check{h}} \geq V$ so that $V=\left(\psi^{h}\right)^{\check{h}}$ using (7). The characterization of $\left(\phi^{\check{h}}\right)^{h}$ is proved in the same way.

Definition 3 1) Define, for all $x \in \Omega$ :

$$
\partial^{h} \psi(x):=\left\{y \in Y: \psi\left(x^{\prime}\right)-\psi(x) \geq h\left(x^{\prime}, y\right)-h(x, y), \text { for all } x^{\prime} \in \Omega\right\}
$$

$\partial^{h} \psi(x)$ is called the h-subdifferential of $\psi$ at $x$, and $\psi$ is h-subdifferentiable at $x$ if and only if $\partial^{h} \psi(x) \neq \emptyset$.

2) Define, for all $y \in Y$ :

$$
\partial^{\breve{h}} \phi(y):=\left\{x \in \bar{\Omega}: \phi\left(y^{\prime}\right)-\phi(y) \geq h\left(x, y^{\prime}\right)-h(x, y) \text {, for all } y^{\prime} \in Y\right\} \text {. }
$$

Note that $\partial^{h} \psi(x)$ and $\partial^{\check{h}} \phi(y)$ can also be defined by:

$$
\partial^{h} \psi(x)=\left\{y \in Y: \psi(x)+\psi^{h}(y)=h(x, y)\right\}
$$

and:

$$
\partial^{\breve{h}} \phi(y)=\left\{x \in \bar{\Omega}: \phi^{\check{h}}(x)+\phi(y)=h(x, y)\right\} .
$$

In particular, if $\psi$ is $h$-convex and $x \in \Omega$, then $y \in \partial^{h} \psi(x)$ if only if $x \in \partial^{\check{h}} \psi^{h}(y)$.

Proposition 2 Let $\psi$ be h-convex and finite, the following assertions hold true:

1) For all $x \in \Omega, \partial^{h} \psi(x)$ is nonempty and compact, and the restriction of the set-valued map $\partial^{h} \psi$ to every closed subset of $\Omega$ has a closed graph,

2) $\psi \in W_{\text {loc }}^{1, \infty}(\Omega)$, and : $\operatorname{esssup}_{\omega}|\nabla \psi| \leq c(\omega)$ for all $\omega \subset \subset \Omega$ and $c(\omega)$ is given by (2),

3) if $\psi$ is differentiable at $x \in \Omega$ and $y \in \partial^{h} \psi(x)$ then

$$
\nabla \psi(x)=\frac{\partial h}{\partial x}(x, y)
$$

4) there exists some Borel map $s_{\psi}$ such that for almost every $x \in \Omega$, $\partial^{h} \psi(x)=\left\{s_{\psi}(x)\right\}$ and $s_{\psi}(x) \in \partial^{h} \psi(x)$ for all $x \in \Omega$. 


\section{Proof.}

Let $A$ be some nonempty subset of $Y \times \mathbb{R}$ such that:

$$
\psi(x)=\sup _{(y, t) \in A} h(x, y)+t, \text { for all } x \in \Omega .
$$

1) Fix $x \in \Omega$ and let $\left(y_{n}, t_{n}\right)$ be some sequence of $A$ such that $h\left(x, y_{n}\right)+t_{n} \rightarrow$ $\psi(x)$ as $n \rightarrow+\infty$. Up to a subsequence we may assume that $y_{n}$ converges to some $\bar{y} \in Y$ so that $t_{n} \rightarrow \bar{t}:=\psi(x)-h(x, \bar{y})$. Let us show now that $\bar{y} \in \partial^{h} \psi(x)$. Let $x^{\prime} \in \Omega$ for all $n, \psi\left(x^{\prime}\right) \geq h\left(x^{\prime}, y_{n}\right)+t_{n}$ passing to the limit we get $\psi\left(x^{\prime}\right) \geq \psi(x)+h\left(x^{\prime}, \bar{y}\right)-h(x, \bar{y})$ i.e. $\bar{y} \in \partial^{h} \psi(x) ; \partial^{h} \psi(x)$ is clearly compact since $Y$ is and $h$ is continuous. The fact that the restriction of $\partial^{h} \psi$ to every closed subset of $\Omega$ has a closed graph is straightforward.

2) Let $\omega \subset \subset \Omega$ and

$$
c(\omega):=\sup _{\left(x_{1}, x_{2}, y\right) \in \omega^{2} \times Y, x_{1} \neq x_{2}}\left|h\left(x_{1}, y\right)-h\left(x_{2}, y\right)\right| \cdot\left\|x_{1}-x_{2}\right\|^{-1}<+\infty .
$$

Let $\left(x_{1}, x_{2}\right) \in \omega^{2}$ we have:

$$
\begin{gathered}
\psi\left(x_{1}\right)=\sup _{(y, t) \in A} h\left(x_{1}, y\right)+t=\sup _{(y, t) \in A} h\left(x_{2}, y\right)+t+h\left(x_{1}, y\right)-h\left(x_{2}, y\right) \\
\leq \psi\left(x_{2}\right)+c(\omega)\left\|x_{1}-x_{2}\right\|
\end{gathered}
$$

finally, reversing order of $x_{1}$ and $x_{2}$ yields the desired result.

3) Let $x \in \Omega$ be a point of differentiabilty of $\psi$ and $y \in \partial^{h} \psi(x)$, let $k \in \mathbb{R}^{n}$, and $t \neq 0$ be such that $[x-t k, x+t k] \subset \Omega$ :

$$
\begin{aligned}
\psi(x+t k)-\psi(x)= & t \nabla \psi(x) \cdot k+o(t) \geq h(x+t k, y)-h(x, y) \\
& =t \frac{\partial h}{\partial x}(x, y) \cdot k+o(t)
\end{aligned}
$$

dividing by $t>0$ and letting $t \rightarrow 0^{+}$in the previous yields

$$
\nabla \psi(x) \cdot k \geq \frac{\partial h}{\partial x}(x, y) \cdot k
$$

similarly the converse inequality also holds taking $t \rightarrow 0^{-}$and since $k$ is arbitrary we get:

$$
\nabla \psi(x)=\frac{\partial h}{\partial x}(x, y)
$$

4) By 2) and Rademacher's Theorem $\psi$ is differentiable a.e. in $\Omega$. On the other, hand since $Y$ is compact and separable and using 1$), \partial^{h} \psi$ admits 
a measurable selection say $s_{\psi}$ (see [6] or the measurable selection Theorem of Brown and Purves in [25]). If $\psi$ is differentiable at $x \in \Omega$ and $y \in \partial^{h} \psi(x)$ then by 3) we get:

$$
\frac{\partial h}{\partial x}(x, y)=\frac{\partial h}{\partial x}\left(x, s_{\psi}(x)\right)
$$

so that with $(3) \partial^{h} \psi(x)=\left\{s_{\psi}(x)\right\}$.

Corollary 1 Let $\psi_{1}$ and $\psi_{2}$ be $h$-convex and finite, if for $\mu$-a.e. $x \in \Omega$

$$
\partial^{h} \psi_{1}(x) \cap \partial^{h} \psi_{2}(x) \neq \emptyset
$$

then $\psi_{1}-\psi_{2}$ is constant.

\section{Proof.}

Using Proposition 2 , we get $\nabla\left(\psi_{1}-\psi_{2}\right)=0$ a.e. in $\Omega$ hence the desired result, since $\Omega$ is connected.

We end this section by a result which will play a crucial role in the proof of the main result. The next Proposition is actually a straightforward generalization of a result of Gangbo [9] which was an important tool in [9] to prove Brenier's Theorem.

Proposition 3 Let $\phi$ be $\check{h}$-convex and finite, let $f \in C^{0}(Y, \mathbb{R})$, define:

$$
\psi_{0}:=\phi^{\check{h}}
$$

and for all $r \in(-1,1)$

$$
\psi_{r}:=(\phi+r f)^{\check{h}}
$$

then also define $A:=\left\{x \in \Omega: \psi_{0}\right.$ is differentiable at $\left.x\right\}$ and $s:=s_{\psi_{0}}$ as in Proposition 2, 4) then, for all $x \in A$ :

$$
\lim _{r \rightarrow 0} \frac{1}{r}\left[\psi_{r}(x)-\psi_{0}(x)\right]=-f(s(x)) .
$$

Since $\mu(\bar{\Omega} \backslash A)=0$, (9) is satisfied a.e. in $\bar{\Omega}$. 


\section{Proof.}

Let $x \in A$, first we have:

$$
\psi_{0}(x)=h(x, s(x))-\phi(s(x))
$$

And, for all $r \in(-1,1)$ :

$$
\psi_{r}(x)=h\left(x, y_{r}\right)-\phi\left(y_{r}\right)-r f\left(y_{r}\right) \text { for all } y_{r} \in \partial^{h} \psi_{r}(x) .
$$

Let $r_{n}$ be some sequence of $(-1,1) \backslash\{0\}$ which converges to 0 and relabel some sequence $y_{r_{n}} \in \partial^{h} \psi_{r_{n}}(x)$ into $y_{n}$.

\section{Step 1.}

Let us show first that $y_{n} \rightarrow s(x)$ as $n \rightarrow+\infty$.

Up to a subsequence we may assume that $y_{n}$ converges to $y \in Y$. First note that:

$$
\left\|\psi_{r_{n}}-\psi_{0}\right\|_{\infty} \leq r_{n}\|f\|_{\infty} \rightarrow 0
$$

and:

$$
\psi_{r_{n}}(x)=h\left(x, y_{n}\right)-\phi\left(y_{n}\right)-r_{n} f\left(y_{n}\right) .
$$

Since $\phi$ is l.s.c., we get:

$$
\underline{\lim }_{n} \phi\left(y_{n}\right) \geq \phi(y)
$$

so that passing to the limit in $(13) \psi_{0}(x) \leq h(x, y)-\phi(y)=h(x, y)-\psi_{0}^{h}(y)$ and then $y \in \partial^{h} \psi_{0}(x)=\{s(x)\}, s(x)$ is therefore the only cluster point of $y_{n}$ so that the whole sequence converges to $s(x)$.

\section{Step 2.}

First, we have:

$$
\frac{1}{r_{n}}\left[\psi_{r_{n}}(x)-\psi_{0}(x)\right]=\frac{1}{r_{n}}\left[\left(h\left(x, y_{n}\right)-\phi\left(y_{n}\right)\right)-(h(x, s(x))-\phi(s(x))]-f\left(y_{n}\right) .\right.
$$

On the one hand:

$$
h\left(x, y_{n}\right)-\phi\left(y_{n}\right) \leq h(x, s(x))-\phi(s(x))
$$

on the other hand:

$$
h\left(x, y_{n}\right)-\phi\left(y_{n}\right) \geq h(x, s(x))-\phi(s(x))+r_{n}\left[f\left(y_{n}\right)-f(s(x))\right]
$$

using (15), (16) and the fact that $y_{n}$ converges to $s(x)$ and passing to the limit in (14) we obtain:

$$
\lim _{n} \frac{1}{r_{n}}\left[\psi_{r_{n}}(x)-\psi_{0}(x)\right]=-f(s(x))
$$


since (17) holds for any sequence $\left(r_{n}\right) \in((-1,1) \backslash\{0\})^{\mathbb{N}}$ that converges to 0 we finally get:

$$
\lim _{r \rightarrow 0} \frac{1}{r}\left[\psi_{r}(x)-\psi_{0}(x)\right]=-f(s(x)) .
$$

\section{Proof of the main statement}

We are now ready to prove Theorem 1 . First note that one obviously has:

$$
\sup (\mathcal{M K}) \geq \sup (\mathcal{M})
$$

Let $(\psi, \phi) \in E_{h}$ and $\gamma \in \Gamma(\mu, \nu)$, one has:

$$
\begin{gathered}
L(\psi, \phi)=\int_{\Omega \times Y}(\psi(x)+\phi(y)) d \gamma(x, y) \\
\geq \int_{\Omega \times Y} h(x, y) d \gamma(x, y)=K(\gamma)
\end{gathered}
$$

so that:

$$
\inf (\mathcal{D}) \geq \sup (\mathcal{M K})
$$

Remark. If $(\psi, \phi) \in E_{h}$ and $s \in \Delta(\mu, \nu)$ (respectively $\left.\gamma \in \Gamma(\mu, \nu)\right)$ are such that $J(s)=L(\psi, \phi)$ (respectively $K(\gamma)=L(\psi, \phi))$ then $(\psi, \phi)$ is a solution of $(\mathcal{D})$ and $s$ is a solution of $(\mathcal{M})$ (respectively $\gamma$ is a solution of $(\mathcal{M K})$ ). -

The first step of the proof is:

Lemma 1 There exists a solution $(\bar{\psi}, \bar{\phi})$ of $(\mathcal{D})$, moreover if $(\psi, \phi)$ is a solution of $(\mathcal{D})$ then $\psi$ is $h$-convex, $\phi$ is $\breve{h}$-convex and those functions are conjugate to each other: $\phi=\psi^{h} \nu$-a.e. and $\psi=\phi^{\check{h}} \mu$-a.e..

\section{Proof.}

Note first that it is clear from $(20)$ that the value of $(\mathcal{D})$ is finite.

\section{Step 3.}

We first prove that if $(\psi, \phi) \in E_{h}$ is a solution of $(\mathcal{D})$ then:

$$
\mu\left(\left\{\psi>\left(\psi^{h}\right)^{\check{h}}\right\}\right)=\mu\left(\left\{\psi>\phi^{\check{h}}\right\}\right)=\nu\left(\left\{\phi>\left(\phi^{\check{h}}\right)^{h}\right\}\right)=0
$$


If $(\psi, \phi) \in E_{h}$ then obviously $\psi \geq \phi^{\check{h}}$ and $\phi \geq \psi^{h}$. Let $\widetilde{\psi}:=\phi^{\check{h}}$ and $\widetilde{\phi}:=\widetilde{\psi}^{h}=\left(\phi^{\breve{h}}\right)^{h}$, by Young's inequality $(\widetilde{\psi}, \widetilde{\phi}) \in E_{h}$ and $\widetilde{\psi} \leq \psi$ and $\widetilde{\phi} \leq \phi$ so that $L(\psi, \phi) \geq L(\widetilde{\psi}, \widetilde{\phi})$. Hence if $(\psi, \phi) \in E_{h}$ is a solution of $(\mathcal{D})$ then:

$$
\nu\left(\left\{\phi>\left(\phi^{\check{h}}\right)^{h}\right\}\right)=0
$$

and

$$
\mu\left(\left\{\psi>\phi^{\check{h}}\right\}\right)=0
$$

this also implies $\mu\left(\left\{\psi>\left(\psi^{h}\right)^{\check{h}}\right\}\right)=0$ since $\left(\psi^{h}\right)^{\check{h}} \geq \phi^{\check{h}}$ and (21) is proved.

\section{Step 4.}

We now prove existence. Let $\left(\psi_{n}, \phi_{n}\right) \in E_{h}^{\mathbb{N}}$ be some minimizing sequence of $(\mathcal{D})$, noting that $L\left(\psi_{n}+a, \phi_{n}-a\right)=L\left(\psi_{n}, \phi_{n}\right)$ and using (21), we may assume with no loss of generality that $\psi_{n}=\phi_{n}^{\breve{h}}, \phi_{n}=\psi_{n}^{h}$ and:

$$
\inf _{Y} \phi_{n}=0
$$

also note that the infimum in (22) is attained since $\phi_{n}$ is l.s.c. say at some point $z_{n}$. Since $\phi_{n} \geq 0$ we get first:

$$
\psi_{n} \leq \max _{\bar{\Omega} \times Y} h
$$

and since $\phi_{n}\left(z_{n}\right)=0$ :

$$
\psi_{n} \geq h\left(., z_{n}\right) \geq \frac{\min }{\Omega} \times Y
$$

so that $\psi_{n}(x)$ is bounded uniformly in $n$ and $x \in \Omega$. On the other hand, using the fact that $\psi_{n}$ is $h$-convex and Proposition 2, assertion 2), we get that $\psi_{n}$ is locally Lipschitz uniformly in $n$. Using Ascoli's Theorem, we may assume, up to a subsequence that $\psi_{n}$ converges uniformly on compact subsets of $\Omega$ to some bounded and locally Lipschitz function $\bar{\psi}$.

\section{Step 5.}

Let us prove that $\bar{\psi}$ is itself $h$-convex. Define, for all $x \in \Omega$ :

$$
\widetilde{\psi}(x):=\sup _{x^{\prime} \in \Omega, y^{\prime} \in F\left(x^{\prime}\right)} \bar{\psi}\left(x^{\prime}\right)+h\left(x, y^{\prime}\right)-h\left(x^{\prime}, y^{\prime}\right)
$$

with:

$$
F\left(x^{\prime}\right):=\bigcap_{N \geq 1} \overline{\bigcup_{n \geq N} \partial^{h} \psi_{n}\left(x^{\prime}\right)}
$$

note that $F\left(x^{\prime}\right) \neq \emptyset$ for $\partial^{h} \psi_{n}\left(x^{\prime}\right)$ is nonempty and compact for all $n$. 
$\widetilde{\psi}$ is clearly $h$-convex and $\widetilde{\psi} \geq \bar{\psi}$ let us show the converse inequality: let $x^{\prime} \in \Omega$ and $y^{\prime}=\lim _{N} y_{n_{N}}$ where $n_{N} \rightarrow+\infty$ and $y_{n_{N}} \in \partial^{h} \psi_{n_{N}}\left(x^{\prime}\right)$, passing to the limit in:

$$
\psi_{n_{N}}(x) \geq \psi_{n_{N}}\left(x^{\prime}\right)+h\left(x, y_{n_{N}}\right)-h\left(x^{\prime}, y_{n_{N}}\right)
$$

we get:

$$
\bar{\psi}(x) \geq \bar{\psi}\left(x^{\prime}\right)+h\left(x, y^{\prime}\right)-h\left(x^{\prime}, y^{\prime}\right)
$$

taking the supremum in the previous finally proves $\bar{\psi}=\widetilde{\psi}$ so that $\bar{\psi}$ is $h$ convex.

\section{Step 6.}

Let $\bar{\phi}:=\bar{\psi}^{h}$ (so that $(\bar{\psi}, \bar{\phi}) \in E_{h}$ ) and let us prove that $(\bar{\psi}, \bar{\phi})$ is a solution of $(\mathcal{D})$. Lebesgue's dominated convergence Theorem yields first:

$$
\int_{\Omega} \psi_{n} d \mu \rightarrow \int_{\Omega} \bar{\psi} d \mu
$$

Now since, for all $(x, y) \in \Omega \times Y, \phi_{n}(y) \geq h(x, y)-\psi_{n}(x)$ we get:

$$
\underline{\lim }_{n} \phi_{n} \geq \bar{\psi}^{h}=\bar{\phi}
$$

using (24) and Fatou's Lemma we get:

$$
\underline{\lim }_{n} \int_{Y} \phi_{n} d \nu \geq \int_{Y} \bar{\phi} d \nu
$$

By $(23)$ and $(25)$ we deduce that $(\bar{\psi}, \bar{\phi})$ is a solution of $(\mathcal{D})$ with $\bar{\psi}=\bar{\phi}^{\breve{h}}$ since $\bar{\phi}=\bar{\psi}^{h}$ and $\bar{\psi}$ is $h$-convex.

The precise duality relations between $(\mathcal{D})$ and $(\mathcal{M}),(\mathcal{M K})$ are given by:

Lemma 2 Let $(\bar{\psi}, \bar{\phi})$ be as in the previous Lemma and $\bar{s}$ be any Borel selection of $\partial^{h} \bar{\psi}$, the following assertions hold:

1) $\bar{s} \in \Delta(\mu, \nu)$ and it is a solution of $(\mathcal{M})$,

2) $\bar{\gamma}:=(i d, \bar{s}) \sharp \mu \in \Gamma(\mu, \nu)$ and it is a solution of $(\mathcal{M K})$

3) $(\mathcal{D})$ is dual to $(\mathcal{M})$ and $(\mathcal{M K})$ in the sense:

$$
\bar{v}:=\inf (\mathcal{D})=\sup (\mathcal{M})=\sup (\mathcal{M K}) .
$$


Proof. Since $\left(\phi^{\check{h}}, \phi\right) \in E_{h}$ for all $\phi, \bar{\phi}$ minimizes $\phi \mapsto \widetilde{L}(\phi):=L\left(\phi^{\check{h}}, \phi\right)$ say for instance in $L^{\infty}\left(Y, \mathcal{B}_{Y}, \nu\right)$.

In particular for all $f \in C^{0}(Y, \mathbb{R})$ :

$$
\begin{gathered}
\lim _{r \rightarrow 0^{+}} \frac{1}{r}[\widetilde{L}(\bar{\phi}+r f)-\widetilde{L}(\bar{\phi})] \geq 0 \\
\frac{1}{r}[\widetilde{L}(\bar{\phi}+r f)-\widetilde{L}(\bar{\phi})]=\int_{Y} f d \nu+\int_{\Omega} \frac{1}{r}\left[(\bar{\phi}+r f)^{\check{h}}-\bar{\phi}^{\check{h}}\right] d \mu
\end{gathered}
$$

Proposition 3, yields first:

$$
\lim \frac{1}{r}\left[(\bar{\phi}+r f)^{\check{h}}(x)-\bar{\phi}^{\check{h}}(x)\right]=-f(\bar{s}(x)), \mu \text {-a.e. }
$$

on the other hand:

$$
\left|\frac{1}{r}\left[(\bar{\phi}+r f)^{\check{h}}-\bar{\phi}^{\check{h}}\right]\right| \leq\|f\|_{\infty}
$$

(26), (27), (28) and Lebesgue's Dominated Convergence Theorem yield then:

$$
\int_{Y} f d \nu-\int_{\Omega} f(\bar{s}(x)) d \mu(x) \geq 0
$$

and the converse inequality obviously holds changing $f$ into $-f$. To sum up, we have proved:

$$
\int_{Y} f d \nu=\int_{\Omega} f(\bar{s}(x)) d \mu(x)
$$

for all $f \in C^{0}(Y, \mathbb{R})$ so that $\nu=\bar{s} \sharp \mu$. In other words $\bar{s} \in \Delta(\mu, \nu)$ and $\bar{\gamma}:=(i d, \bar{s}) \sharp \mu \in \Gamma(\mu, \nu)$. Now note that since $\bar{\psi}(x)+\bar{\phi}(\bar{s}(x))=h(x, \bar{s}(x))$ and using $\bar{s} \sharp \mu=\nu$ we have:

$$
\begin{gathered}
L(\bar{\psi}, \bar{\phi})=\inf (\mathcal{D})=\int_{\Omega}[\bar{\psi}(x)+\bar{\phi}(\bar{s}(x))] d \mu(x) \\
=\int_{\Omega} h(x, \bar{s}(x)) d \mu(x)=J(\bar{s})=K(\bar{\gamma})
\end{gathered}
$$

Finally, using (20) we get:

$$
J(\bar{s})=\sup (\mathcal{M})=K(\bar{\gamma})=\sup (\mathcal{M K})=\inf (\mathcal{D})
$$

which proves that $\bar{s}$ is a solution of $(\mathcal{M})$ and $\bar{\gamma}$ is a solution of $(\mathcal{M K})$, hence the desired result. 
The last thing to prove is uniqueness:

Lemma 3 Let $(\bar{\psi}, \bar{\phi}), \bar{s}$ and $\bar{\gamma}$ be as in the previous Lemma, the following assertions hold:

1) if $(\psi, \phi)$ is a solution of $(\mathcal{D})$ then there exists a constant a such that:

$$
\begin{gathered}
\psi-\bar{\psi}=a, \mu \text {-a.e. } \\
\phi-\bar{\phi}=-a, \nu \text {-a.e. }
\end{gathered}
$$

2) if $s$ is a solution of $(\mathcal{M})$ then $s=\bar{s} \mu$-a.e.,

3) $\bar{\gamma}:=(i d, \bar{s}) \sharp \mu$ is the unique solution of $(\mathcal{M K})$.

\section{Proof.}

1) Assume that $(\psi, \phi)$ is a solution of $(\mathcal{D})$ then, using Lemma 1 , we may assume that

$$
\psi=\phi^{\check{h}} \text { and } \phi=\psi^{h}
$$

let $s$ be some Borel selection of $\partial^{h} \psi$. We already know that $s$ is a solution of $(\mathcal{M})$ by Lemma 2. Young's inequality yields:

$$
\bar{\psi}(x)+\bar{\phi}(s(x)) \geq h(x, s(x)), \text { for all } x \in \Omega
$$

using $J(s)=L(\bar{\psi}, \bar{\phi})$ and the fact that $s \in \Delta(\mu, \nu)$ we get:

$$
L(\bar{\psi}, \bar{\phi})=\int_{\Omega}[\bar{\psi}(x)+\bar{\phi}(s(x))] d \mu(x)=\int_{\Omega} h(x, s(x)) d \mu(x)
$$

(33) and (32) yield:

$$
\bar{\psi}(x)+\bar{\phi}(s(x))=h(x, s(x)) \text {, for } \mu \text {-almost every } x \in \Omega
$$

or equivalently $s(x) \in \partial^{h} \bar{\psi}(x)$ a.e..

Finally, Corollary 1 implies that there exists some constant $a$

$$
\psi-\bar{\psi}=a, \mu \text {-a.e. and } \phi-\bar{\phi}=-a \nu \text {-a.e. }
$$

2) Similarly, if $s$ is a solution of $(\mathcal{M})$ then

$$
\bar{\psi}(x)+\bar{\phi}(s(x))=h(x, s(x)) \text {, for } \mu \text {-almost every } x \in \Omega
$$

and then $s=\bar{s} \mu$-a.e. 
3) Let $\gamma$ be a solution of $(\mathcal{M K})$ so that:

$$
K(\gamma)=\int_{\Omega \times Y} h d \gamma=L(\bar{\psi}, \bar{\phi})=\int_{\Omega \times Y}[\bar{\psi}(x)+\bar{\phi}(y)] d \gamma(x, y)
$$

since $(\bar{\psi}, \bar{\phi}) \in E_{h}$ we get:

$$
h(x, y)=\bar{\psi}(x)+\bar{\phi}(y), \gamma \text {-a.e. }
$$

Let $G(\bar{s})$ be the graph of $\bar{s}$ and $G\left(\partial^{h} \bar{\psi}\right)$ be that of $\partial^{h} \bar{\psi},(35)$ implies:

$$
\gamma(G(\bar{s}))=\gamma\left(G\left(\partial^{h} \bar{\psi}\right)\right)=1 .
$$

Let $A$ be some Borel subset of $\Omega$ and $B$ be some Borel subset of $Y$, by (36), we get:

$$
\gamma(A \times B)=\gamma(A \times B \cap G(\bar{s}))
$$

using (36) once again, we get then:

$$
\gamma(A \times B)=\gamma\left(\left(A \cap \bar{s}^{-1}(B)\right) \times Y\right)
$$

and since $\pi_{1} \sharp \gamma=\mu$ :

$$
\gamma(A \times B)=\mu\left(A \cap \bar{s}^{-1}(B)\right)=\bar{\gamma}(A \times B)
$$

so that $\gamma=\bar{\gamma}$ which ends the proof.

We end this section by a Polar-Factorization type consequence of the main result:

Corollary 2 Up to $\mu$-a.e. equivalence there exists a unique Borel map $s$ such that:

1) there exists some $h$-convex potential $\psi$ such that $s(x) \in \partial^{h} \psi(x)$ for all $x \in \Omega$,

2) $s$ pushes forward $\mu$ through $\nu$.

Proof. $\bar{s}$ defined as previously, satifies the desired result, now, if $s$ satisfies 1) and 2) then

$$
J(s)=L\left(\psi, \psi^{h}\right) \geq \inf (\mathcal{D})=\sup (\mathcal{M})
$$

so that, using Lemma $3, s=\bar{s} \mu$-a.e. 


\section{Economic application and generalized Spence- Mirrlees condition}

We end this article by an application of our result to the theory of incentives. More precisely, we are going to prove a re-allocation principle that generalizes a well-known one in dimension 1.

Assume that agents' preferences are given by the quasi-linear utility function:

$$
V(x, y, t)=h(x, y)+t,
$$

where $x \in \Omega$ is the agent's type or parameter, $y \in Y$ is an action and $t \in \mathbb{R}$ is some monetary compensatory transfer. We make the same assumptions on $\Omega, Y, h$ and $\mu$ as previously. Note that in this case, the probability measure $\mu$ captures the distribution of types among agents.

A key concept in that theory is that of incentive-compatible contracts:

Definition 4 1) A contract is a pair of functions $(s, t): \Omega \rightarrow Y \times \mathbb{R}$. by:

2) The potential associated with a contract $(s, t)$ is the function $V_{s, t}$ defined

$$
V_{s, t}(x):=h(x, s(x))+t(x) \text { for all } x \in \Omega .
$$

3) The contract $(s, t)$ is incentive-compatible if and only if:

$$
h(x, s(x))+t(x) \geq h\left(x, s\left(x^{\prime}\right)\right)+t\left(x^{\prime}\right), \text { for } \operatorname{all}\left(x, x^{\prime}\right) \in \Omega^{2} .
$$

4) A function $s: \Omega \rightarrow Y$ is implementable if and only if there exists $t$ : $\Omega \rightarrow \mathbb{R}$ such that $(s, t)$ is incentive-compatible.

Remark. The incentive-compatibility condition (37) means that it is optimal for every agent to announce his true parameter.

\subsection{The usual Spence-Mirrlees condition and the one dimensional case}

In the special one-dimensional case i.e. $\Omega=(a, b), Y=[\alpha, \beta]$ and under the assumption that $h$ is of class $C^{2}$ and satisfies the Spence-Mirrlees condition:

$$
\frac{\partial^{2} h}{\partial x \partial y}>0
$$

then we have the standard characterization result (see [17], [24], [21]): 
Proposition $4 s$ is implementable if and only if $s$ is nondecreasing.

Remark. Note that Spence-Mirrlees condition (38) implies our assumption (3) on $h$.

In this one-dimensional case and under assumption (38), we also have the other characterization:

Lemma 4 The following assertions are equivalent:

1) $s$ is nondecreasing

2) there exists $\psi$ h-convex such that $s(x) \in \partial^{h} \psi(x)$ for all $x \in \Omega$.

Proof. First assume that $s$ is nondecreasing and define:

$$
\psi(x):=\int_{a}^{x} \frac{\partial h}{\partial x}(t, s(t)) d t
$$

we are going to prove that $\psi$ is $h$-convex and $s(x) \in \partial^{h} \psi(x)$ for all $x$. Define for all $x$ :

$$
\widetilde{\psi}(x):=\sup _{x^{\prime} \in \Omega} \psi\left(x^{\prime}\right)+h\left(x, s\left(x^{\prime}\right)\right)-h\left(x^{\prime}, s\left(x^{\prime}\right)\right)
$$

$\widetilde{\psi}$ is $h$-convex and $\widetilde{\psi} \geq \psi$, let us show that $\psi \geq \widetilde{\psi}$. Let $x^{\prime} \in \Omega$, we have:

$$
\begin{gathered}
\psi(x)-\psi\left(x^{\prime}\right)-h\left(x, s\left(x^{\prime}\right)\right)+h\left(x^{\prime}, s\left(x^{\prime}\right)\right) \\
=\int_{x}^{x^{\prime}}\left[\frac{\partial h}{\partial x}\left(t, s\left(x^{\prime}\right)\right)-\frac{\partial h}{\partial x}(t, s(t))\right] d t
\end{gathered}
$$

and the latter is nonnegative using the fact that $s$ is nondecreasing and (38). This yields $\psi=\widetilde{\psi}$ so that $\psi$ is $h$-convex and the previous computation also yields $s(x) \in \partial^{h} \psi(x)$.

Conversely assume that for all $x, s(x) \in \partial^{h} \psi(x)$ for some $h$-convex $\psi$. We have:

$$
\psi\left(x^{\prime}\right)-\psi(x) \geq h\left(x^{\prime}, s(x)\right)-h(x, s(x))
$$

and

$$
\psi(x)-\psi\left(x^{\prime}\right) \geq h\left(x, s\left(x^{\prime}\right)\right)-h\left(x^{\prime}, s\left(x^{\prime}\right)\right)
$$

so that:

$$
h\left(x^{\prime}, s(x)\right)-h(x, s(x))+h\left(x, s\left(x^{\prime}\right)\right)-h\left(x^{\prime}, s\left(x^{\prime}\right)\right) \leq 0
$$

or equivalently:

$$
\int_{x}^{x^{\prime}}\left[\frac{\partial h}{\partial x}(t, s(x))-\frac{\partial h}{\partial x}\left(t, s\left(x^{\prime}\right)\right)\right] d t \leq 0
$$


note finally that, with (38), the previous expression has the sign of $\left(x^{\prime}-\right.$ $x)\left(s(x)-s\left(x^{\prime}\right)\right)$ so that $s$ is nondecreasing.

The previous characterizations can be viewed equivalently as a re-allocation principle via monotone rearrangements:

Proposition 5 Let $s_{0}$ be some Borel map : $\Omega \rightarrow Y$ and let $\widetilde{s}$ be the non decreasing rearrangement of $s_{0}$ with respect to $\mu$ then $\widetilde{s}$ is the only Borel map which satisfies:

1) $\widetilde{s}$ is implementable,

2) $\widetilde{s}$ and $s_{0}$ are equimeasurable i.e. : $\widetilde{s} \sharp \mu=s_{0} \sharp \mu$.

For properties of monotone rearrangements see [18]. Recall that $\widetilde{s}$ is defined as follows; first define:

$$
F_{s_{0}}(y):=\mu\left(\left\{s_{0}<y\right\}\right), \text { for all } y \in Y
$$

then, for all $x \in \Omega$ :

$$
\widetilde{s}(x):=\inf \left\{y \in Y \text { s.t. } F_{s_{0}}(y)>x\right\} .
$$

Remark. $\widetilde{s}$ is the solution of:

$$
\sup _{s \in \Delta\left(\mu, s_{0} \sharp \mu\right)} \int_{\Omega} h(x, s(x)) d \mu(x)
$$

In other words, $\widetilde{s}$ maximizes the average surplus in the class of maps that have the same cumulative function as $s_{0}$.

The previous remark can be viewed as an easy consequence of HardyLittlewood inequality (see [18]).

Let us finally note that if $h$ and $g$ both satisfy (38) then the associated Monge's problems have the same solution. Of course, this result is very specific of the one-dimensional problem.

Proposition 6 Let $h$ and $g$ be two functions from $\Omega \times Y$ to $\mathbb{R}$ of class $C^{2}$ that both satisfy (38); let $\nu$ be some Radon probability measure on $Y$. Then both problems:

$$
\sup _{s \in \Delta(\mu, \nu)} J_{h}(s):=\int_{\Omega} h(x, s(x)) d \mu(x)
$$


and

$$
\sup _{s \in \Delta(\mu, \nu)} J_{g}(s):=\int_{\Omega} g(x, s(x)) d \mu(x)
$$

have the same solution.

Proof. Let $s$ be the maximizer of $J_{h}$ over $\Delta(\mu, \nu)$, from Lemma 4 and Theorem $1, s$ is nondecreasing. There exists then $\psi g$-convex such that $s(x) \in \partial^{g} \psi(x)$ for all $x$ and since $s \sharp \mu=\nu, s$ maximizes $J_{g}$ over $\Delta(\mu, \nu)$.

\subsection{Re-allocation principle in the general case}

Our aim now is to consider the general problem where $\Omega$ is a bounded connected open subset of $\mathbb{R}^{n}$, and $\mu$ is some probability measure in $\Omega$ which is absolutely continuous with a positive Radon-Nikodym derivative with respect to the $n$-dimensional Lebesgue measure, and such that $\mu(\partial \Omega)=0, Y$ is a compact Polish space and $h$ satisfies (1), (2) and (3).

We shall prove a similar re-allocation principle as in the one dimensional case so that (3) is a natural generalization of the Spence-Mirrlees condition. A first attempt was made by Mc Afee and Mc Millan in [15] to characterize incentive-compatibility in a multi-dimensional setting, the condition of these authors is much stronger than (3) and their characterization requires $s$ to be differentiable which is of course not required in what follows since $Y$ need not have a linear structure.

We start by a characterization result that can be found in [5]

Proposition 7 Let $s: \Omega \rightarrow Y$ and $t: \Omega \rightarrow \mathbb{R}$ then we have:

1) $(s, t)$ is incentive-compatible if and only if

$$
\begin{gathered}
V_{s, t} \text { is } h \text {-convex, and } \\
s(x) \in \partial^{h} V_{s, t}(x) \text { for all } x \in \Omega .
\end{gathered}
$$

2) $s$ is implementable if and only if there exists some $h$-convex function $\psi$ such that:

$$
s(x) \in \partial^{h} \psi(x) \text { for all } x \in \Omega \text {. }
$$

Then the re-allocation principle can be stated as: 
Theorem 2 Let $s_{0}$ be an arbitrary Borel function $\Omega \rightarrow Y$, there exists a unique (up to a.e. equivalence) Borel map $\bar{s}$ such that:

1) $\bar{s}$ is implementable,

2) $s_{0}$ and $\bar{s}$ are equimeasurable i.e. $\bar{s} \sharp \mu=s_{0} \sharp \mu$.

Moreover, $\bar{s}$ is the solution of the Monge's Problem:

$$
\sup _{s \in \Delta\left(\mu, s_{0} \sharp \mu\right)} \int_{\Omega} h(x, s(x)) d \mu(x) .
$$

Proof. Proof follows directly from Theorem 1 and Proposition 7.

Remark. The economic interpretation of this result is the following : any allocation plan can be rearranged into some implementable one in a unique way ; $\bar{s}$ is therefore in some sense a monotone rearrangement of $s_{0}$ and it is obtained by maximizing the average surplus in the set of measure-preserving maps $\Delta\left(\mu, s_{0} \sharp \mu\right)$.

Moreover, at least from a theoretical point of view, one can use our main result to find a tarif $\bar{t}$ such that the pair $(\bar{s}, \bar{t})$ is incentive compatible. Let $(\bar{\psi}, \bar{\phi})$ be a solution of the dual problem of $(39)$, and define for all $x \in \Omega$ :

$$
\bar{t}(x):=-\bar{\phi}(\bar{s}(x))=-h(x, \bar{s}(x))+\bar{\psi}(x),
$$

then it can be checked easily that the pair $(\bar{s}, \bar{t})$ is an incentive-compatible contract, let us indeed consider a pair of types $\left(x, x^{\prime}\right)$, we have:

$$
\begin{aligned}
h(x, \bar{s}(x))+\bar{t}(x) & =\bar{\psi}(x)=\bar{\phi}^{\check{h}}(x) \geq h\left(x, \bar{s}\left(x^{\prime}\right)\right)-\bar{\phi}\left(\bar{s}\left(x^{\prime}\right)\right) \\
& =h\left(x, \bar{s}\left(x^{\prime}\right)\right)+\bar{t}\left(x^{\prime}\right) .
\end{aligned}
$$

\section{References}

[1] A.D. Aleksandrov. Existence and uniqueness of a convex surface with a given integral curvature, C.R. (Doklady) Acad. Sci. URSS, vol. 35 (1942).

[2] J.D. Benamou, Y. Brenier. A numerical method for the Optimal TimeContinuous Mass Transport Problem and related problems, Contemporary Mathematics, vol. 226 (1999). 
[3] Y. Brenier. Polar Factorization and monotone rearrangements of vector valued functions, Communications in Pure and Applied Mathematics, vol. 44 (1991).

[4] G. Bouchitté, G. Buttazzo, P. Seppecher. Shape Optimization solutions via Monge-Kantorovich equation, C.R. Acad. Sci. Paris (1998).

[5] G. Carlier. A general existence result for the principal-agent problem with adverse selection, Journal of Mathematical Economics, Vol. 35, pp. 129-150 (2001).

[6] C. Castaing, M. Valadier. Convex analysis and measurable multifunctions, Lecture Notes in Mathematics, vol. 580, Springer-Verlag (1977).

[7] P. Choné. Étude de quelques problèmes variationnels intervenant en géométrie riemannienne et en économie mathématique, $\mathrm{PhD}$ Thesis, Univ. Toulouse I (1999).

[8] I. Ekeland, R. Temam. Convex Analysis and Variational Problems, North-Holland (1972).

[9] W. Gangbo. An elementary proof of the polar factorization of vectorvalued functions, Arch. Rational Mech. Anal., vol. 128 (1994).

[10] W. Gangbo, R.J. Mc Cann. The Geometry of Optimal Transportation, Acta Math., vol. 177 (1996).

[11] L.V. Kantorovich, G.P. Akilov. Functional Analysis, Pergamon Press, Oxford (1982).

[12] L.V. Kantorovich, G.S. Rubinstein. On a functional space and certain extremal problems, Dokl. Akad. Nauk. SSSR, vol. 115 (1957).

[13] V. Levin. Abstract cyclical monotonicity and Monge solutions for the general Monge-Kantorovich Problem, Set-Valued Analysis, vol. 7 (1999).

[14] V. Levin. Reduced cost functions and their applications, Journal of Mathematical Economics, vol. 28 (1997).

[15] R.P. Mc Afee, J. Mc Millan Multidimensional Incentive Compatibility and Mechanism Design, Journal of Economic Theory, vol. 46 (1988).

[16] R.J. Mc Cann. Existence and uniqueness of monotone measurepreserving maps, Duke Math. Journal, vol. 80 (1995). 
[17] J. Mirrlees. Optimal Tax Theory : a synthesis, Journal of Public Economics, vol 7 (1976).

[18] J. Mossino. Inégalités isopérimétriques et applications en physique, Hermann, Paris (1984).

[19] S.T. Rachev. The Monge-Kantorovich mass transference problem and its stochastic applications, Theory Proba. Appl., vol. 29 (1984).

[20] S.T. Rachev, L. Rüschendorf. Mass Transportation Problems. Vol. I: Theory; Vol. II : Applications, Springer-Verlag (1998).

[21] J.-C. Rochet. A necessary and Sufficient Condition for Rationalizability in a Quasi-linear context, Journal of Mathematical Economics, vol. 16 (1987).

[22] J.-C. Rochet, P. Choné. Ironing, Sweeping and Multidimensional screening, Econometrica, vol. 66 (1998), pp. 783-826.

[23] R. T. Rockafellar. Convex Analysis, Princeton University Press (1970).

[24] M. Spence. Competitive and optimal responses to signals, Journal of Economic Theory, vol. 7 (1974).

[25] D. H. Wagner. Survey of measurable selection theorems : an update, in Measure Theory, Lecture Notes in Mathematics, vol. 794, SpringerVerlag (1979). 